\title{
Development and validation of the Leuven intelligibility sentence test with male speaker (LIST-m).
}

For the final published version, please refer to:

Jansen S., Koning R., Wouters J. \& van Wieringen A. 2013. Development and validation of the Leuven intelligibility sentence test with male speaker (LIST-m). International journal of audiology, [epub ahead of print].

Sofie JANSEN ${ }^{1}$, Raphael KONING ${ }^{1}$, Jan WOUTERS ${ }^{1}$, \& Astrid VAN WIERINGEN ${ }^{1}$

${ }^{1} \mathrm{KU}$ Leuven - University of Leuven, Department of Neurosciences, ExpORL, Leuven, Belgium

Key words:

Speech intelligibility, sentence test, cochlear implant

\section{Corresponding author:}

Sofie Jansen, ExpORL, KU Leuven

Herestraat 49, bus 721

B-3000 Leuven

BELGIUM

Tel: $\quad+3216330477$

Sofie.Jansen@med.kuleuven.be 


\begin{abstract}
Objective: In addition to the LIST with a female speaker (van Wieringen \& Wouters, 2008), a new speech perception test with a male voice was developed and validated, for evaluating the intelligibility performance of cochlear implant ( $\mathrm{Cl}-$ ) users or severely hearing impaired persons. Design: Three experimental steps were carried out: (1) a perceptual optimization of the recorded materials, (2) an evaluation in normal hearing ( $\mathrm{NH}$ ) listeners, and (3) a validation in $\mathrm{Cl}$-users. Measurements were performed both in quiet and in noise. Study sample: Forty-four NH subjects and $6 \mathrm{Cl}$-users participated. Results: After selecting the sentences with a similar intelligibility, the reference psychometric curve for $\mathrm{NH}$ listeners was determined, showing steep slopes for measurements in quiet $(12.3 \% / \mathrm{dB})$ and in noise $(18.7 \% / \mathrm{dB})$, similar to the LIST with female voice. The 38 lists of 10 sentences yielded equal scores, and the within-subject test-retest reliability was high (1.7 dB in quiet, $1.1 \mathrm{~dB}$ in noise). For the $\mathrm{Cl}$-users, parallel psychometric curves were found between the LIST with male and female voice. Conclusions: The LIST-m is a reliable and valid speech intelligibility test that can be used for $\mathrm{Cl}$-users, both in quiet and in noise.
\end{abstract}

\title{
Introduction
}

Speech intelligibility tests based on everyday sentences presented in quiet or noise have proven their great value in audiological research (e.g. evaluation of speech enhancement algorithms in cochlear implant ( $\mathrm{Cl}$ ) processors) as well as in clinical audiology (e.g. quantification of hearing aid benefit). Due to the fact that complex speech stimuli representative for real-world communication are used, there is a need for speech tests in many different languages. Furthermore, for experiments on informational masking or stream segregation, the availability of a large set of sentences for different speakers of a different gender is required.

For the Dutch language, the Leuven Intelligibility Sentence Test (LIST, van Wieringen \& Wouters, 2008) was developed complementary to the already existing tests of Plomp \& Mimpen (1979a) and Versfeld et al (2000). Special for the LIST is the lower speech rate at which the sentences are being uttered ( 2.5 syllables per second versus 4.7 for the Versfeld sentences (van Wieringen \& Wouters, 2008)), and the possibility to determine key word scores. Only in this way, it is possible to test most $\mathrm{Cl}$-users or persons with a severe hearing loss for whom standard speech tests are often too difficult. In addition to the LIST with a female voice, there is a need for a similar speech material with a male voice: LIST-m.

This article describes the development and validation of the LIST-m which was carried out in three steps. The first step concerns the optimization in which the speech reception threshold (SRT, i.e. the level at which an intelligibility score of $50 \%$ is obtained) is determined for each recorded sentence, so that a subset of sentences with a similar SRT can be selected and divided in lists of 10. In the second step, the evaluation, the list equivalency, the reference psychometric curve, and the testretest reliability is determined in normal hearing $(\mathrm{NH})$ listeners, so that it can be compared to the normative values reported for the original LIST with female speaker (LIST-f). Finally, in the validation, 
results for both the LIST-m and LIST-f are compared in Cl-users. All the applied procedures as described in this article, are in accordance with the procedures that have been used for the development of LIST-f.

\section{Material and Methods}

\section{Preparation of speech and noise material}

\section{Recording of sentences}

As described in detail in van Wieringen and Wouters (2008), a large set of 730 everyday sentences had been selected for the LIST. In each sentence, the key words were determined. In general, these are all words except prepositions, articles, pronouns, conjunctions, and some of the auxiliary verbs. The number of key words per sentence ranged from 2 to 7 , with an average and median of 3. All sentences were formerly recorded by four professional speakers in an anechoic room. The recordings of one of the two male speakers are used in this study. Further details on the recording conditions are given in van Wieringen \& Wouters (2008).

For each sentence, the average root mean square (RMS) level was calculated using frames of $50 \mathrm{~ms}$. Frames with an RMS level below 0.001 (-60 dB FS) were not taken into account, so that the RMS of a sentence was not influenced by silence parts in between words. The average RMS across all 730 sentences equaled 0.0403 (-27.9 dB FS).

\section{Generation of stationary speech-shaped noise}

The long-term average speech spectrum (LTASS) of the 730 sentences was determined as follows: For each sentence, silence parts were removed (frames of $20 \mathrm{~ms}$ with RMS $<0.001$ ) and the spectrum was calculated with a 4096-points fast Fourier transform using a rectangular window and without overlap. These spectra were then averaged, applying a weight according to the length of each sentence. For this LTASS, a 2048-taps finite impulse response filter was generated and applied on an 11 seconds long white noise. Transients at the start and the end of the speech-shaped noise were removed to allow looping of the noise without any clicks. The average RMS level of the noise was $0.0442(-27.1 \mathrm{~dB} F \mathrm{~F})$. The $0.8 \mathrm{~dB}$ difference with the average speech level $(-27.9 \mathrm{~dB} F \mathrm{~F})$ is automatically compensated for in the testing software (cf. below).

\section{Subjects}

In total, $44 \mathrm{NH}$ subjects and $6 \mathrm{Cl}$-users participated in this study. All $\mathrm{NH}$ listeners had pure-tone thresholds below or equal to $15 \mathrm{~dB} \mathrm{HL}$ for all octave frequencies between 250 and $8000 \mathrm{~Hz}$. The 24 $\mathrm{NH}$ subjects in the optimization measurements had an average age of 22, ranging from 18 to 48 years old. The age of the $20 \mathrm{NH}$ participants in the evaluation measurements ranged between 18 and 21 years old. The $\mathrm{Cl}$-subjects, all with the same type of implant by Cochlear Ltd, were between 19 and 69 years old and were all experienced users (> 5 years). One of them was prelingually deaf and received his implant at the age of 2 years. This study was approved by the Medical Ethics 
Committee of the University of Leuven (KU Leuven) and University Hospitals Leuven, and written informed consent was obtained from all participants.

\section{Test setup}

The APEX 3 software (Francart et al, 2008) was used to present the stimuli, automatically mixing the speech and the noise to the desired signal-to-noise ratio (SNR), and to store the results for each trial. When testing the $\mathrm{NH}$ subjects, the signals were sent out through a 24-bit sound card (LynxOne, Lynx Studio Technology) and a headphone buffer (HB-7, Tucker-Davis Technologies) to a pair of headphones (HDA200, Sennheiser). The stationary speech-shaped noise was used to calibrate the setup at $80 \mathrm{~dB}$ SPL, measured through an artificial ear (type 4153, Brüell \& Kjær). Participants of the optimization measurements were tested in a sound-proof booth or quiet room. For the evaluation measurements, all tests were conducted in a double-walled sound-proof booth. For the $\mathrm{Cl}$-users, an experimental L34 processor, provided by Cochlear Ltd, was used for direct transmission of the stimuli to the subject's implant. The $\mathrm{Cl}$-measurements were conducted in a quiet room.

\section{Procedures}

\section{Optimization}

Twenty-four NH subjects listened to all 730 sentences at $-10,-8,-6$, or $-4 \mathrm{~dB}$ SNR, so that each sentence was presented to 6 listeners per SNR. The signals were presented monaurally to the subject's best ear. The level of the noise was held constant at $65 \mathrm{~dB}$ SPL. The noise started and stopped $500 \mathrm{~ms}$ before and after each sentence. The listeners were instructed to repeat the sentences as accurately and as completely as possible. A sentence score based on key words was determined. This means that a score of 1 is given when all key words in a sentence are understood correctly, otherwise the score is set to 0 . The order of the sentences and SNRs was randomized.

\section{Evaluation}

Based on the optimization results, a subset of sentences was selected and divided in 39 lists of 10 . These lists were then all presented to $20 \mathrm{NH}$ participants using the 1-up 1-down adaptive procedure with a fixed step size of $2 \mathrm{~dB}$. The first sentence in a list was repeated with increasing speech level until it was understood correctly. After scoring the last sentence in a list, the SNR of the (imaginary) $11^{\text {th }}$ sentence was determined. The SRT was then estimated by averaging the last 6 SNRs. Again, sentence scoring based on key words was applied.

Half of the subjects conducted all test lists in noise, which was presented at a constant level of $65 \mathrm{~dB}$ SPL. The first sentence in a list started at $-12 \mathrm{~dB}$ SNR. Here as well, the noise started and ended 500 ms before and after a sentence. The other $10 \mathrm{NH}$ subjects were tested in quiet. Now, the first sentence in a list was presented at $18 \mathrm{~dB}$ SPL. In both conditions, the signals were presented monaurally to the subject's best ear. The presentation order of the lists was randomized.

\section{Validation}

In a last step, 10 lists of LIST-m and 10 lists of LIST-f were presented to $6 \mathrm{Cl}$-users. Intelligibility scores for both speakers were determined in 5 conditions (quiet, $+10,+6,+2$, and $-2 \mathrm{~dB}$ SNR) in a test-retest 
design. The presentation order of the conditions was always from high to low SNR, the speaker order within a condition was randomized. Test lists were chosen randomly, but care was taken that an individual sentence was never presented twice to the same listener. Both sentence scores based on key words and key word scores were determined. In all 5 conditions, the speech level was fixed at 65 $\mathrm{dB}$ SPL. Before the start of the test, each listener performed one training list of LIST-f in quiet.

\section{Results}

\section{Optimization}

For each of the 730 recorded sentences, the SRT was estimated by means of a logistic regression on the intelligibility scores at the four presented SNRs. In order to select a set of sentences with a similar intelligibility, the following exclusion procedure was followed. First, 135 sentences with an SRT below -10 or above $-4 \mathrm{~dB}$ SNR were excluded. Given the range of presented SNRs, the error on the SRT estimation was also rather high for most of these sentences. In a second step, sentences with an SRT deviating by more than 1 standard deviation from the mean SRT were excluded (the mean SRT across the 595 sentences remaining after step 1 equaled $-7.5 \mathrm{~dB}$ SNR with a standard deviation of $1.5 \mathrm{~dB}$ ). In this way, a set of 395 homogeneous sentences was obtained.

Similar to the LIST-f, lists of 10 sentences were constructed with an equal number of syllables (89 to 90 ) and key words ( 32 to 33). In this regard, the 5 sentences with a very low number of syllables (less than 4) or a very high number of syllables (more than 16) were further removed. Of the 390 finally selected sentences of LIST-m there were 193 sentences which do not occur in the final speech material of LIST-f. For practical reasons, 190 of these 'unique' sentences were put together and form the first 19 test lists.

\section{Evaluation}

List equivalency

In order to examine the list equivalency, a repeated-measures analysis of variance (ANOVA) was conducted. A significant effect of test list on the SRT was found, both for measurements in noise $[F(7.2,65.2)=2.565 ; \quad p=0.020]$ and in quiet $[F(6.9,61.8)=3.149 ; p=0.007]$ (Greenhouse-Geisser correction). Pairwise comparisons (with Bonferroni correction) revealed that one list yielded significantly lower (better) SRTs in quiet than two other lists did. Therefore, it was decided to remove this list from the final test material. The final version of the LIST with male voice thus consists of 38 test lists, of which 19 are unique lists. The maximal deviation of a list-specific SRT from the overall mean is $1.2 \mathrm{~dB}$ in noise and $1.7 \mathrm{~dB}$ in quiet.

\section{Norm values and reference psychometric function}

Subject-specific SRTs were obtained by averaging the SRTs of the 38 final test lists. Based on the mean and the standard deviation across the 10 participants per condition, the reference SRT for NH listeners can be determined. In noise, the reference SRT was -7.8 $( \pm 0.4) \mathrm{dB}$ SNR. In quiet, it was 21.1 $( \pm 2.5) \mathrm{dB}$ SPL. A detailed overview of the subject-specific results is given in Table 1 . 
Table 1. Evaluation results in $\mathrm{NH}$ listeners: overview of the subject-specific results in noise and in quiet (SD = standard deviation; SE = standard error on the parameter estimate). SRTs in noise are expressed in dB SNR, SRTs in quiet in dB SPL.

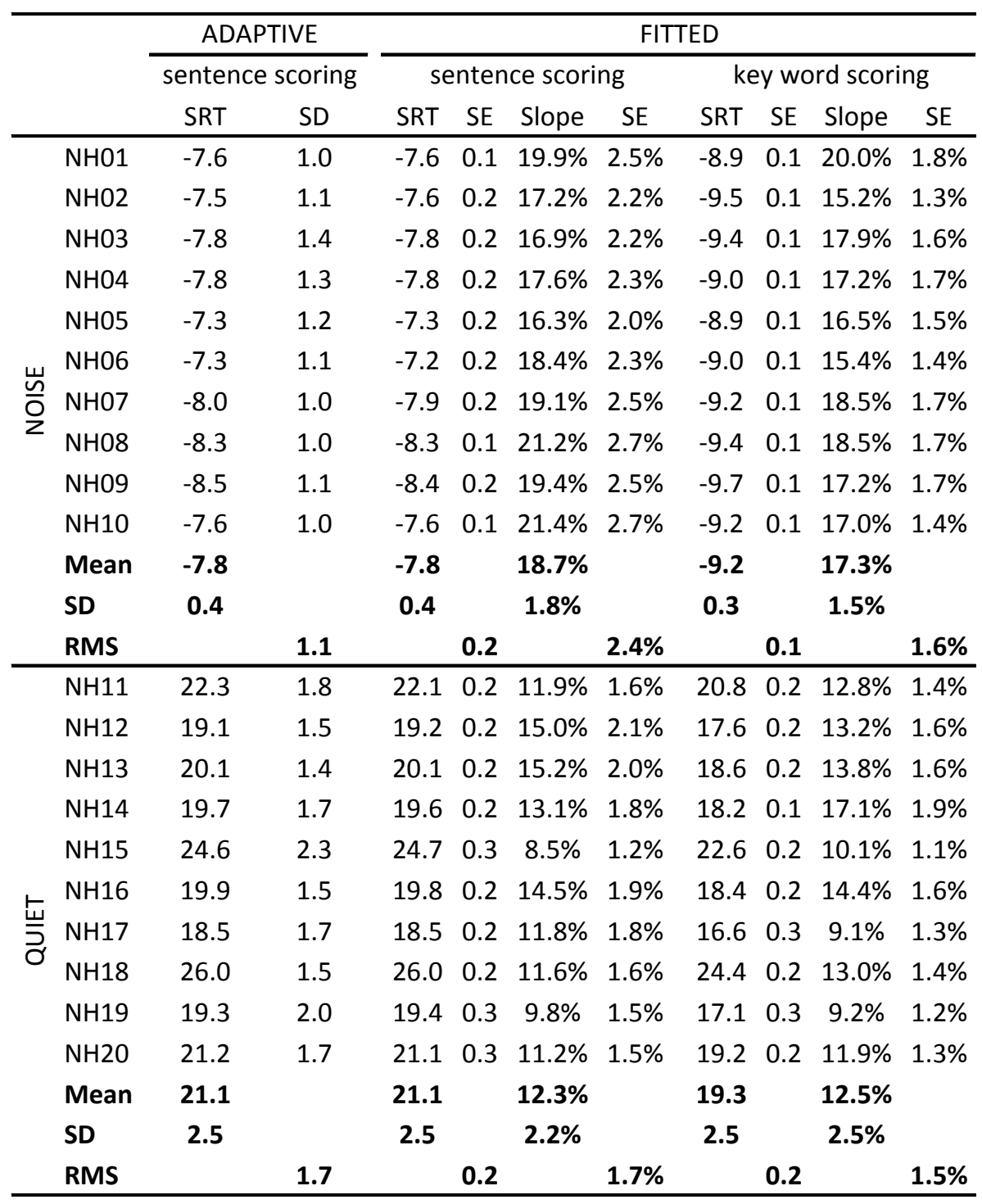

Besides the adaptively determined SRT, the complete psychometric function was estimated for each subject as well. For each sentence presented to a listener, the score (0 or 1 ) and the corresponding SNR were available. In case the first sentence within a list was presented more than once, only the first presentation was taken into account. Based on these 380 combinations of SNR and score, a logistic regression fit could be applied on the data of each listener. Table 1 summarizes the subjectspecific SRT and slope estimates and their standard error. Besides the reference SRT, which corresponds exactly to the adaptively determined reference SRT, the reference slope can be determined as well: $18.7( \pm 1.8) \% / \mathrm{dB}$ in noise and $12.3( \pm 2.2) \% / \mathrm{dB}$ in quiet.

Although the simple up-down adaptive procedure requires sentence scoring, key word scores were stored as well. Similarly to the above described fitting procedure, SRT and slope estimates were thus determined using key word scores as well (see Table 1): the reference SRT is $-9.2( \pm 0.3) \mathrm{dB}$ SNR for 
noise and $19.3( \pm 2.5) \mathrm{dB}$ SPL in quiet, and reference slope is $17.3( \pm 1.5) \% / \mathrm{dB}$ in noise and $12.5( \pm 2.5)$ $\% / \mathrm{dB}$ in quiet.

\section{Test-retest reliability}

The test-retest reliability can be defined as the quadratic mean of the within-subject standard deviations of repeatedly measured adaptive SRTs. Based on the 38 lists presented to each subject, the reliability of LIST-m is estimated at $1.1 \mathrm{~dB}$ in noise and $1.7 \mathrm{~dB}$ in quiet (see Table 1). It needs to be noted that this reliability is of course interrelated to the list equivalency reported above.

\section{Validation}

For the $6 \mathrm{Cl}$-users, test and retest intelligibility scores were determined in 5 conditions for both LIST$\mathrm{m}$ and LIST-f. Figure 1 shows both the key word (KW) and sentence (S) scores, averaged over test and retest. Parallel curves could be drawn for the two different speakers. A repeated-measures ANOVA on the RAU transformed scores (Rationalized Arcsine Units; Studebaker, 1985) revealed that there were no interaction effects between any of the three factors 'test-retest', 'speaker', and 'condition' ( $p>0.05)$. There was also no main effect of 'test-retest' $[F(1,5)=0.051 ; p=0.831]$, nor for 'speaker' $[F(1,5)=0.649 ; p=0.457]$. The only significant effect was the main effect of 'condition' $[F(4,20)=71.459 ; p<0.001]$, where higher SNRs yielded higher speech intelligibility scores.

As a measure of test-retest reliability, Intraclass correlation coefficients (ICC) were determined on the test and retest intelligibility scores of the six subjects in the five conditions (two-way random model, absolute agreement, single measures). For both speakers, a strong agreement was found for key word scoring ( 0.92 for female and 0.91 for male speaker), and for sentence scoring ( 0.85 for female and 0.92 for male speaker). This strong agreement is also shown in the test-retest scatter plots of Figure 2 .

\section{Discussion}

Compared to the results of LIST-f, the new speech material with male speaker yields highly similar results. For the measurements in noise, the test-retest reliability of $1.1 \mathrm{~dB}$, the reference SRT of -7.8 $d B$ SNR, and the reference slope of $18.7 \% / d B$ are very close to the values for LIST-f $(1.17 \mathrm{~dB},-8.0 \mathrm{~dB}$ $\mathrm{SNR}$, and $17.8 \% / \mathrm{dB}$, respectively), as reported by van Wieringen and Wouters (2008). A sentence-innoise test with a reliability around $1 \mathrm{~dB}$ and a slope above $15 \% / \mathrm{dB}$ is generally considered an accurate test (Plomp \& Mimpen, 1979; Nilsson et al, 1994; Versfeld et al, 2000), suitable both in a clinical setting as well as for research. Furthermore, although the sentences were not optimized for key word scoring, nor for testing in quiet, a steep psychometric curve could yet be found in these conditions as well.

Only one difference occurred between the results of the two speakers of the LIST: the reference SRT in quiet for LIST-m lies significantly lower than the norm values reported for LIST-f (21.1 versus 27.1 $\mathrm{dB} S \mathrm{SL}$ ). Therefore, 4 of our $10 \mathrm{NH}$ listeners tested in quiet, additionally listened to two lists of the female speaker. Even within the same subjects, using the same test setup and calibration method, 
this difference remained. It can be concluded that this is a pure effect of certain speaker characteristics, which disappears when both speech materials are presented in their own speechshaped noise.

Also for $\mathrm{Cl}$-users the LIST-m proves to yield reliable and valid results. Test and retest scores showed a low variability and intelligibility scores were highly similar to the LIST-f. Due to the low speech rate of 2.3 syllables per second (similar to the 2.5 syllables per second of LIST-f) and the possibility to determine key word scores, the LIST-m is, as the LIST-f, expected to be suitable for most Cl-users or severely hearing impaired listeners who are not able to reach meaningful results on standard sentence-in-noise tests.
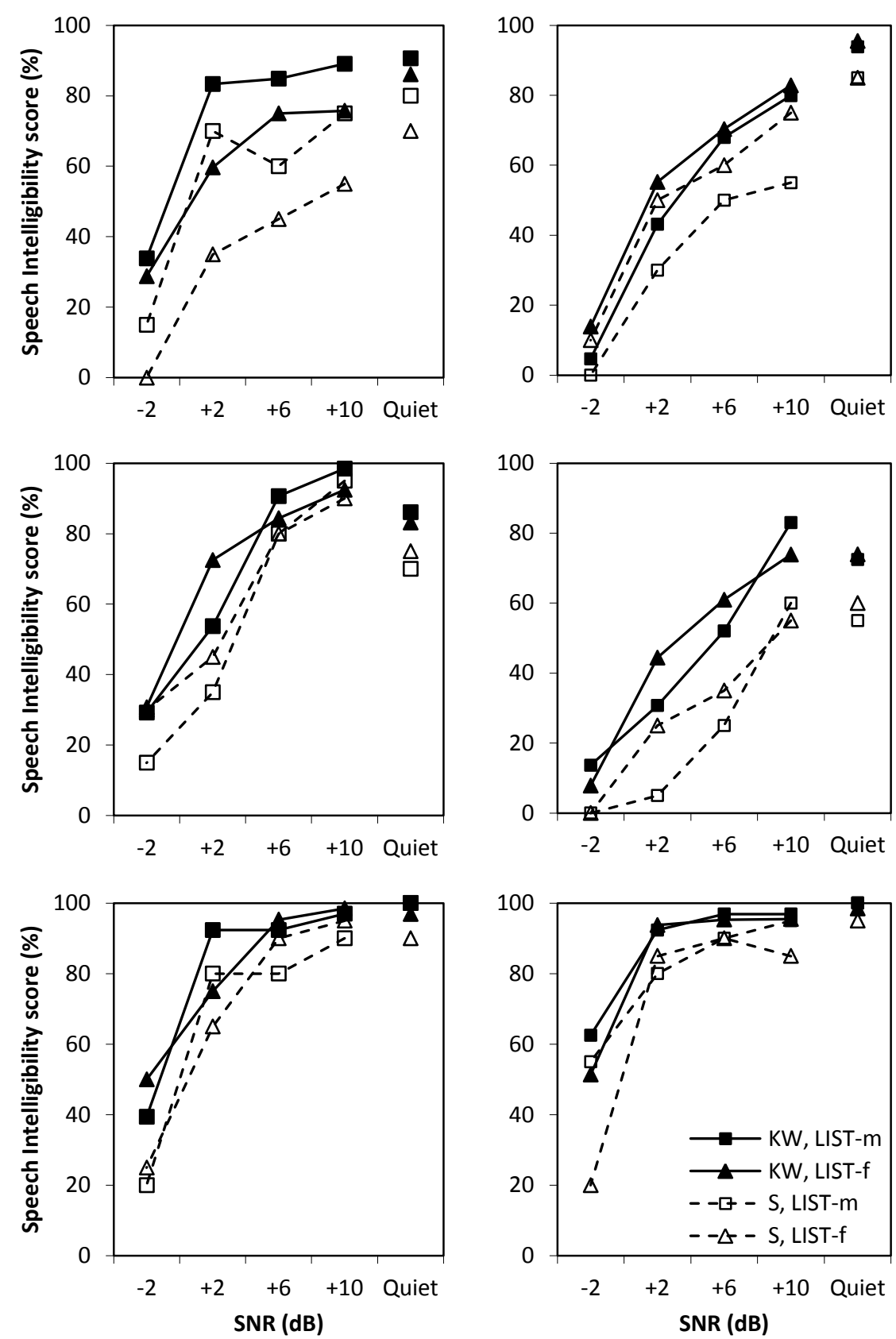

Figure 1. Validation results in $\mathrm{Cl}$-users: Intelligibility scores at 5 conditions (quiet, $+10,+6,+2$, and $-2 \mathrm{~dB}$ SNR) for LIST-m (squares) and LIST-f (triangles), applying key word scores (KW, filled symbols) and sentence scores (S, open symbols). Each panel shows the scores of one $\mathrm{Cl}$-subject. 

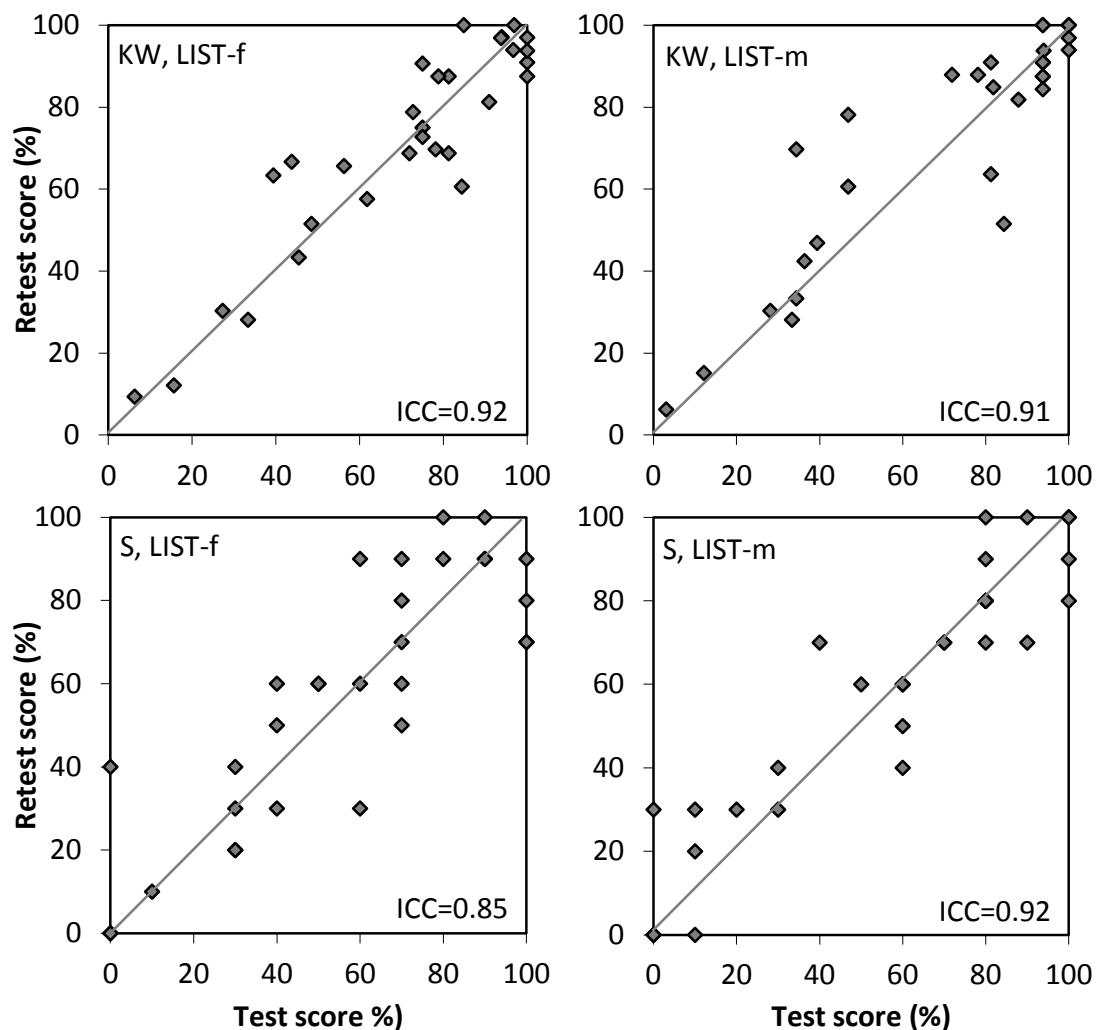

Figure 2. Scatter plots of the test versus retest scores for the female speaker (LIST-f, left panels) and male speaker (LIST$\mathrm{m}$, right panels), and for key word scores (KW, upper panels) and sentence scores ( $\mathrm{S}$, lower panels). Symbols represent the scores for the six subjects in the five test conditions. The grey line is the identity line $(y=x)$. Intraclass correlation coefficients (ICC) are given per panel.

\section{Conclusions}

This study shows that the LIST-m is a reliable and valid speech intelligibility test that can be used for $\mathrm{Cl}$-users, both in quiet and in noise. Additionally to the 35 test lists of the original LIST with female speaker, 38 equivalent test lists are now available with a male speaker.

The LIST-m material can be obtained from the Research Group ExpORL through the following website: http://kuleuven.be/expORL.

\section{Acknowledgements}

The authors would like to thank Griet Mertens, Liesbeth Verschoren, Joke De Schutter, and Mieke Hermans for administering the optimization and evaluation measurements. Heleen Luts and Tom Francart are gratefully acknowledged for their technical and methodological support. 


\section{References}

Francart T., van Wieringen A. \& Wouters J. 2008. APEX 3: a multi-purpose test platform for auditory psychophysical experiments. J. Neurosci. Methods, 172, 283-93.

Nilsson M., Soli S.D. \& Sullivan J.A. 1994. Development of the Hearing In Noise Test for the measurement of speech reception thresholds in quiet and in noise. J. Acoust. Soc. Am., 95, 1085-99.

Plomp R. \& Mimpen A.M. 1979. Improving the reliability of testing the speech reception threshold for sentences. Audiol. Off. organ Int. Soc. Audiol., 18, 43-52.

Studebaker G.A. 1985. A “rationalized” arcsine transform. J. Speech Hear. Res., 28, 455-62.

Van Wieringen A. \& Wouters J. 2008. LIST and LINT: sentences and numbers for quantifying speech understanding in severely impaired listeners for Flanders and the Netherlands. Int. J. Audiol., 47, 348-55.

Versfeld N.J., Daalder L., Festen J.M. \& Houtgast T. 2000. Method for the selection of sentence materials for efficient measurement of the speech reception threshold. J. Acoust. Soc. Am., 107, 1671-84. 